\title{
ASSOCIATION BETWEEN LEUKOCYTE AND HEMORRHAGIC TRANSFORMATION AFTER ISCHEMIC STROKE
}

\author{
Quhong Song, Chenchen Wei, Yannan wang, Ming Liu \\ Department of Neurology, West China Hospital of Sichuan \\ University, Stroke Clinical Research Unit, Chengdu, China
}

\section{Background:}

Leukocyte infiltrated into the ischemic tissue after ischemic stroke is associated with blood-brain-barrier damage and may cause hemorrhagic transformation (HT). Whether leukocyte is associated with HT after ischemic stroke and whether the association depends on stroke etiology remains an open question.

\begin{abstract}
Methods:
Acute ischemic stroke patients within 7 days from stroke onset were included. Blood samples were obtained within 24 hours after admission. HT was defined as hemorrhage presented on follow-up MRI or CT but not on baseline CT. Stroke was divided into five categories according to the criteria of the Trial of Org 10172 in Acute Stroke Treatment (TOAST).
\end{abstract}

\section{Results:}

We included 973 patients (mean age $63.94 \pm 14.60$ years; $63 \%$ male). HT occurred in 115 patients (11.82\%). Leukocyte was grouped into quartiles (Quartile 1: $<6.22$, Quartile 2:6.22-7.66, Quartile 3: 7.67-9.31, Quartile 4:>9.31), and Quartile 1 was set as reference. Univariate analysis identified risk of HT was higher in Quartile 3 ( OR 2.205, 95\% CI 1.217-3.994) and Quartile 4 (OR 2.075, 95\% CI 1.140-3.778). In multiple regression analysis, leukocyte were independently associated with HT in all ischemic stroke patients (OR 2.104, 95\% CI 1.080-4.099), and in the subgroup of cardioembolic stroke (OR 4.411,95\% CI 1.372-4.179), but not in the subgroup of the other three types of stroke.

\section{Conclusions:}

Leukocyte counts were significantly associated with the risk of HT in all ischemic stroke patients, especially in cardio-embolic stroke patients.
This research was supported by the National Natural Science Foundation of China (81620108009) and Key Research and Development Program, Science \& Technology Department of Sichuan Province (Grant No. 2017SZ0007). 